Tequio 1(1), 2017: 25-31

ISSN: 2594-0546

\title{
DIMENSIÓN EDUCATIVA EN PROCESOS RITUALES DE COMUNIDADES INDÍGENAS
}

\author{
Gloria Evangelina Ornelas-Tavarez ${ }^{1}$ \\ Fecha de recepción: 4 de noviembre de 2016 \\ Fecha de aceptación: 25 de mayo de 2017
}

\begin{abstract}
Resumen - Con este artículo se pretende abonar al estudio de la diversidad desde la interculturalidad y la educación, argumentando conceptual y metodológicamente la importancia de la cosmovisión mesoamericana en el abordaje del proceso educativo inmerso en los rituales en los que participan miembros de comunidades indígenas, en dos contextos: en la educación familiar-comunitaria indígena mixe y en la educación escolar de niños itinerantes nahuas y otomíes en un pueblo originario del sur de la Cuenca de México, denominado Xochimilco, concretamente en una escuela 9-14. Las investigaciones, cuyos referentes empíricos y análisis conceptuales los lleva a cabo, en el primer caso, Blanca Zitlali López Martínez, tocan temas de identidad, cultura y educación a través de un ritual familiar-comunitario; el segundo caso, a cargo de Tahnee Nemo Ramírez, problematiza aspectos relacionados con la socialización política de niños itinerantes en situación de extra edad en el espacio escolar urbano, entre otros.
\end{abstract}

Palabras clave:

Cosmovisión, educación, pueblos indígenas, ritualidad.
Abstract - This paper summarizes progress in the research project on Education and Ritualism among Indigenous Peoples, approved and financed for 2016-2018 by the Diversity and Interculturality Academic Area of the National University of Education Sciences (UPN)-Ajusco campus. The purpose is to study diversity from a perspective of interculturality and education, arguing conceptually and methodologically the importance of the Mesoamerican world view in research into the educational process immersed in rituals into which members of indigenous communities participate. One of the two contexts under study is Mixe indigenous family-community education. The other context is classroom education for itinerant Nahua and Otomi children in a town of original peoples in the south of the Valley of Mexico, specifically in a school for 9-14 year olds in the Xochimilco district. Both investigations are advancing in their empirical referents and conceptual analyses. The first piece of research is carried out by Blanca Zitlali López Martínez on the topics of identity, culture, and education implemented via a family-community ritual. The second project by Tahnee Nemo Ramírez problematizes aspects related with the political socialization of children under itinerant situation of over age in the urban educational space, within other aspects.

\section{Keywords:}

Worldview, education, rituality, peoples indigenous. 


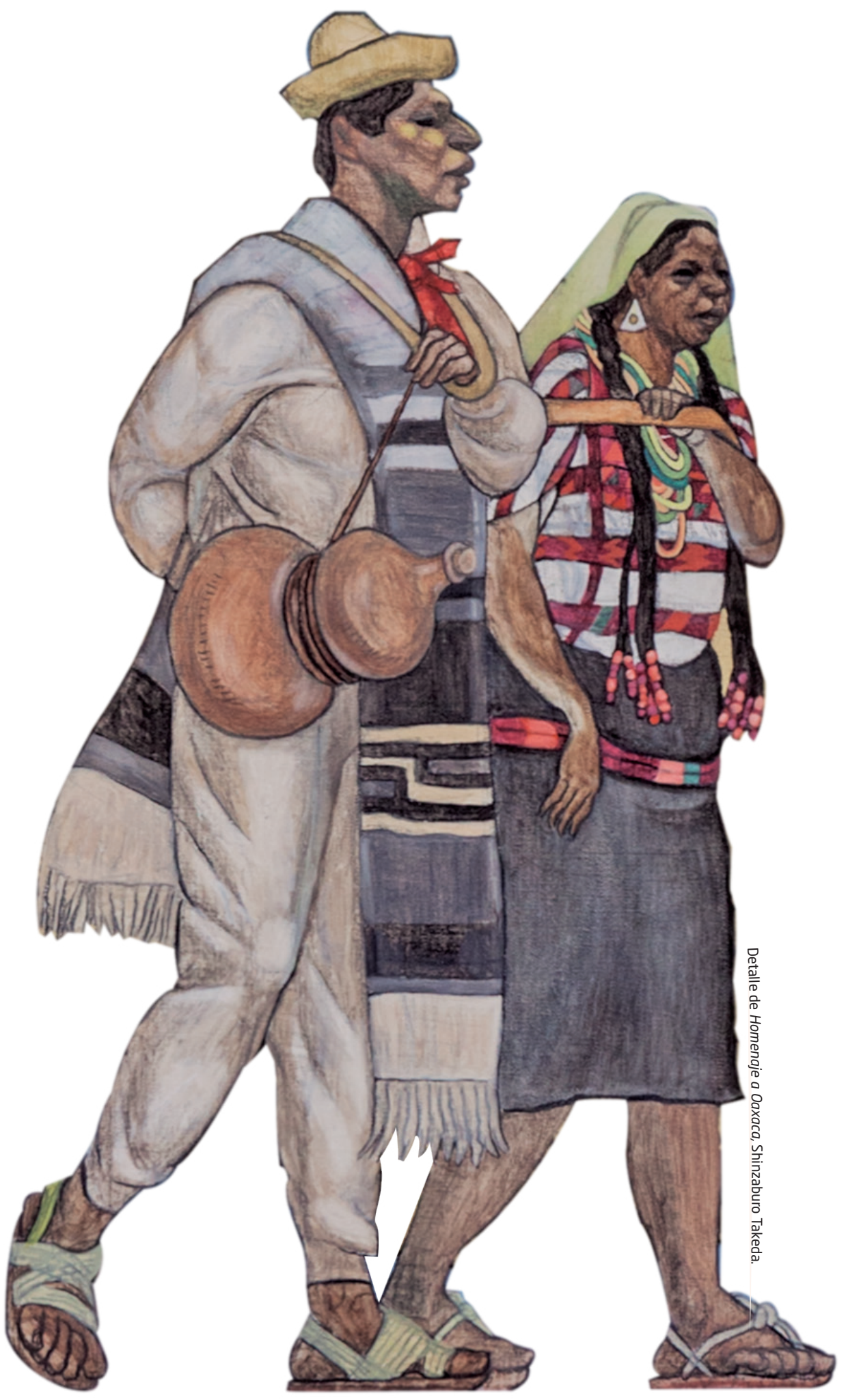

Tequio, septiembre-diciembre 2017, vol. 1, no. 1 


\section{Problema de estudio}

D e acuerdo con Medina (2000, p. 11), Mesoamérica, área cultural a la que pertenecen las comunidades

originarias de los sujetos de estudio, es producto de una compleja y heterogénea dinámica de relaciones sociales que hizo posible la incidencia dialéctica de la historia común y las historias particulares de cada uno de los pueblos que la constituyen, para formar una visión del mundo rica en expresiones regionales y locales que permiten examinar las cosmovisiones mesoamericanas de nuestros días, a pesar de los procesos de colonización, nacionalismo, modernización, transnacionalización y globalización.

Las comunidades indígenas que constituyen dicha área cultural se caracterizan por referentes históricos, espaciales, temporales y simbólicos de larga data, así como por sus núcleos institucionales, compuestos por una organización cívica-religiosa y un ciclo festivo concéntrico y extensivo, basado en lo comunitario, donde la cultura, la cosmovisión, la identidad y la acción política de sus sujetos colectivos/individuales enfrentan la modernidad y el neoliberalismo impuestos en la región latinoamericana.

Desde el punto de vista de Medina (2000, p. 16), la cosmovisión es un espacio privilegiado para reconocer las estructuras de poder articuladas a concepciones de raíz mesoamericana y medieval, y de gran incidencia en la discusión actual acerca de la autonomía, el poder local y las formas propias de gobierno.

Sin embargo, en el ámbito educativo mexicano poco se ha teorizado sobre el concepto de cosmovisión desde un punto de vista antropológico; comúnmente se le refiere como filosofía o ideología, excluyendo, entre otras, las narraciones míticas y los procesos rituales, así como las relaciones entre ambos elementos que la constituyen.

Aunado a ello, por lo general la etnografía educativa utilizada en un país pluricultural como el nuestro no se ha apoyado suficientemente en la investigación sociohistórica y comparativa necesaria, para explicar de manera más profunda los procesos simbólicos que se estudian.

Por otra parte, dada su movilidad espacial, los nuevos miembros de las comunidades originarias a veces no reparan en la vigencia e importancia de rituales que se efectúan dentro de sus familias, o bien, al formar parte de las grandes ciudades y obligarse a permanecer en la escuela, no registran conscientemente las fortalezas de su origen y su ser comunitario; sin embargo, el núcleo duro de la cosmovisión mesoamericana persiste en ellos, a través del efecto educativo que incide en su pensamiento y actuación.

Por todo lo anterior es importante centrar la atención en el proceso educativo, en la función conservadora, transmisora, socializadora y transformadora del ritual que constituye a los sujetos, a su cultura e identidad social e individual, no sin antes explicitar el marco más amplio en el que se inscriben dichos rituales: la cosmovisión mesoamericana.

\section{Preguntas y objetivos}

Las preguntas que guían el presente artículo son: ¿Cuáles son los referentes que permiten explicar la presencia contemporánea de la cosmovisión mesoamericana? ¿Los rituales comunitarios son la vía privilegiada para mostrar elementos prehispánicos en la cosmovisión de grupos indígenas, o de indígenas-urbanos, contemporáneos? ¿Qué papel ostenta la educación en la transmisión, socialización, internalización, adecuación y/o traducción de una explicación primordial, cristalizada en rituales, en el contexto de la acción moderna de los grupos?

Los objetivos que se pretenden alcanzar son los siguientes:

- Conceptualizar la cosmovisión mesoamericana y justificar su presencia actual.

- Argumentar la reproducción del tiempo primordial en las concepciones, ideas y creencias que se viven en rituales llevados a cabo hoy en día.

- Resaltar dimensión educativa de los rituales indígenas en los que se socializa y desde los cuales también se construye la identidad individual/comunitaria. 


\section{Referentes teórico-metodológicos}

Las perspectivas teórico-metodológicas asumidas se desprenden de la etnografía mesoamericana, la cual permite apreciar, parafraseando a Medina (2000, p. 11), la riqueza y originalidad de la antropología mexicana, con una presencia antigua en la cultura nacional, así como su propio desarrollo técnico y temático dentro de una comunidad científica.

El trabajo de campo que la sustenta parte de la caracterización del entorno espacial y temporal, de los pobladores mesoamericanos, sus creencias y prácticas simbólicas, dentro de las cuales se inscriben los ciclos rituales. Se articula en la búsqueda de rasgos comunes y singulares de dicha cosmovisión. Los registros orales, escritos y gráficos, plagados de narrativas y costumbres, muestran formas de pensar y actuar. Dichas narraciones están fuertemente ligadas a los referentes identitarios de estos pueblos, a la personificación del origen, del sentido de la celebración comunitaria y a su relación, defensa o transacción frente a otras concepciones y acciones político-religiosas y educativas.

La cosmovisión es la perspectiva estructurada que va más allá del complejo mundo de las creencias; en ella, los miembros de una comunidad combinan de manera coherente sus nociones y sus prácticas respecto del medio en el que nacieron y socializaron originalmente, el contexto en el cual viven o desde el cual emigraron y sobre el cual sitúan una parte de su vida y la del hombre en general. Para Medina (2000, pp. 11-13), está constituida por la filosofía del ser, la condición humana, la razón del mundo, la organización de la experiencia de la vida, los aspectos existenciales y la explicación del mundo, componentes que los individuos en una sociedad determinada, en condiciones históricas, sociales y culturales específicas, expresan de manera viva y profundamente humana, atados a su tiempo y espacio. Es un sistema de representación simbólica que se vincula con las estructuras sociales y económicas de la sociedad, a través de la ideología que define formas de conciencia y acción específicamente sistematizadas.
La configuración de las ideologías y la reproducción de las cosmovisiones están determinadas por la historia, sus elementos no son estáticos ni se expresan monolíticamente. Su polivalencia funcional se sujeta al cambio histórico, pero estas diversas interpretaciones surgen dentro de un patrón común, al interior de una matriz cultural denominada cosmovisión, parafraseando a Broda (2001, p. 18).

El estudio de la cosmovisión posibilita explorar las múltiples dimensiones del modo en que se percibe culturalmente el entorno, en tanto aspectos constitutivo de las creencias, las prácticas, las instituciones; en tanto vehículo transmisor y educador de las explicaciones del mundo y del lugar del individuo en relación con el universo, conformado por una multiplicidad y diversidad de contenidos que implican una activa participación social.

Para Portal (1997, p. 29), en México "el pensamiento moderno se constituye sobre la base de una cosmovisión ancestral prehispánica que persiste a través de la Ilamada 'tradición' [...], su construcción se fundamenta en formas 'premodernas' de pensamiento, en las cuales la concepción mítica juega un papel determinante".

Por su parte, Broda parafrasea a Báez-Jorge, quien en su obra de 1998 establece:

[...] la vigencia histórica de las creaciones de la conciencia social, así como su difusión, dependen de las adecuaciones y reelaboraciones a que se han sometido [...] los mitos y los rituales tienen papeles primordiales, patentes en las cosmovisiones; [...] representan insustituibles lenguajes que hacen posible comprender la interrelación de los diferentes planos ideológicos y sociales que vinculan el pasado con el presente. Mito y ritual [...] muestran los senderos de la dinámica transcultural y el decurso histórico que, en el caso de los pueblos mesoamericanos, está signado por un largo y conflictivo proceso de resistencia étnica (Broda, 2001, p. 22). 


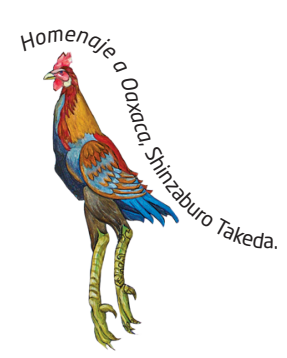

La raíz más profunda de la continuidad mesoamericana se ubica en el trabajo agrícola, en torno a la milpa, donde el maíz es protagónico. En él se sientan las bases de la organización social; las cuestiones de orden técnico y científico se entraman en sistemas de creencias que transmiten experiencias y conocimientos en un contexto sumamente ritualizado. A través de él se construye históricamente la cosmovisión, en la cual conceptos y metáforas remiten a fuerzas y particularidades reconocidas en el trato con la naturaleza, nutriendo las concepciones más generales de tiempo y espacio (Medina, 1997, p. 13).

Así, siguiendo a Medina (2000, p. 18), la cosmovisión mesoamericana se constituye por características de continuidad de las sociedades y culturas del siglo XVI, como un solo modelo para ese siglo y para los pueblos contemporáneos, aunque la diversidad actual permite identificar variantes regionales y abrir problemas significativos con la finalidad de identificar procesos históricos de largo alcance. Por ello, la configuración de la nación es un proceso que involucra al conjunto de los pueblos que componen tanto a la Nueva España como a la sociedad del periodo independiente; cada segmento del territorio mexicano expresa a su manera el devenir histórico de alcance nacional.

El acto fundacional de la organización comunitaria de dichos pueblos, según Medina (2007, pp. 178-179), se estructura política y socialmente con la delimitación de sus tierras, en los paisajes donde se inscriben una historia y una concepción del mundo, sustentada en la documentación oficial de la época colonial y en la designación de un santo patrón con su templo, conjuntamente con el cabildo, el centro y la cabecera comunales. Sobre ella se despliega su persistente lucha contra el despojo de sus tierras y aguas.

Dicha organización responde a una cultura que se cristaliza cuando se distingue de otra, cuando en sus instituciones sociales se observa cierta singularidad y semejanzas; que al ser estudiada a partir de narraciones míticas revela las nociones locales de autoridad y los principios simbólicos que durante siglos la han hecho posible, así como a través de sus prácticas ceremoniales, que legitiman la existencia de una jerarquía comunitaria, de acuerdo con Millán (2007, pp. 21-22).

Los ciclos calendáricos, basados en el ciclo agrícola mesoamericano, se complementan con aquellos festivos comunitarios, como la fiesta del santo patrón que los hermana y enlaza con otros pueblos y sus respectivos santos patrones, constituyéndose como uno de los principales ejes de la cosmovisión que es socializada, transmitida, reproducida y actualizada a través de las familias y los miembros de la comunidad. Este ejercicio educativo se apoya en narrativas míticas y procesos rituales cotidianos, así como en una ritualidad propiamente dicha.

Con la modernidad, la construcción del Estadonación y la historia oficial difundida mediante la educación escolar, estosciclos festivos seentrelazan con el calendario de fiestas y conmemoraciones nacionales que marcan el ritmo y la secuencia de la programación escolar oficial, determinada por la SEP (su presencia en la Cuenca de México dentro de escuelas primarias, una del pueblo de San Andrés Totoltepec, se puede ver en Ornelas, 2005 y 2007; y otra del centro delegacional Tlalpan, en Ornelas, 2007a; así como en un análisis comparativo de ambos casos, en Ornelas, 2010).

En todas estas celebraciones y en cada uno de los ciclos festivos, las familias se involucran entretejiendo también sus propios ciclos de vida y el de cada uno de sus integrantes, muchas de las veces ampliando la parentela extensa con nuevos miembros previamente elegidos como compadres-padrinos. 
Dentro de dichos ciclos, y en este caso, se subrayan los rituales, definidos desde la perspectiva de Turner (1980) como fases de amplios procesos sociales pautados en el tiempo, constituidos por unidades conformadas por objetos simbólicos y aspectos serializados de la conducta simbólica. Algunos de estos rituales corrigen las desviaciones del comportamiento dictado por la costumbre, mientras que otros -denominados periódicos o de las crisis vitales- previenen extravíos y conflictos.

Al igual que los mitos, retomando el punto de vista de López Austin (1996, pp. 335-364), consideramos que los rituales son tarea educativa de los padres o adultos a cargo de los niños, pero también de la comunidad o grupo al que pertenecen. A través de la comunicación y actuación de diversos contenidos, los rituales forjan historias, forman las instituciones, rigen las vidas de los individuos y cumplen sus propósitos; afirman creencias, validan procesos 0 estados naturales o sociales y conforman costumbres y organizaciones. Mantienen viva la tradición, conservan y transforman la memoria, constituyen un núcleo que asimila e incorpora el saber y el actuar nuevo. Ordenan el conocimiento y la práctica, estructurando y clasificando el cosmos, reforzando el saber. Explican, sintetizan justificaciones que el hombre elabora en su acción cotidiana acerca de la sociedad y la naturaleza. Defienden posiciones y apoyan derechos, mismos que pueden ser plurales y contradictorios.

La dimensión educativa de estos rituales se hace imprescindible, no sólo porque enlaza o socializa a las generaciones en la transmisión de valores y conocimientos, sino que además, en su afán de cohesionar, reafirman el carácter común de dichos valores y conocimientos del grupo; legitiman (remitiendo a los tiempos conformadores) la razón de las costumbres, el fundamento de las instituciones, el origen de las divisiones sociales, la fuente de los derechos territoriales, la naturaleza y el comportamiento de las cosas; amén de que regulan las relaciones sociales.
Según Turner (1980, pp. 53-55), cada tipo de ritual puede ser considerado como una configuración de símbolos. El símbolo es una marca, algo que conecta lo desconocido con lo conocido, por lo que el proceso de simbolización hace visibles, audibles y tangibles tanto creencias como ideas, valores, sentimientos y disposiciones psicológicas que no pueden ser directamente percibidos; hace público lo privado, o social lo que es personal.

\section{A manera de conclusión abierta}

En los casos de estudio que se encuentran en proceso de registro y análisis, consideramos que dentro de la ritualística mixe, el ciclo de vida y de rituales de las crisis vitales, es importante definir el de la "purificación" del miembro de la familia elegido, con la finalidad de convertirlo en adulto a través de un trance liminar donde el especialista, los padres y los padrinos -representantes de la comunidad- adquieren una gran responsabilidad social e individual. Constituye un ritual familiar-comunitario propiamente dicho, en comparación con los escolares, en el que es posible identificar distintas cosmovisiones: la nacional, la del sistema educativo, la del establecimiento, la mesoamericana y la comunitaria, tanto en aquellos de la vida cotidiana, así como al interior de rituales en sí mismos, como las ceremonias o los de paso, entre otros, la ceremonia de la bandera o el fin de ciclo escolar.

La comparación entre ellos como procedimiento heurístico permitirá distinguir equivalencias entre episodios, etapas, reconocer personajes y símbolos, encontrar datos complementarios, descubrir significados y asuntos particulares, mismos que al ser acompañados por narrativas míticas, por concepciones, ideas $y / 0$ creencias, así como por referentes teóricos, posibilitarán la emergencia del nodo de la cosmovisión mesoamericana, aun en contextos institucionales estatales, como la escuela primaria.

Con todo lo anterior, apoyamos la reflexión, el análisis y el intercambio de experiencias indagativas relacionadas con la viva presencia del núcleo duro de la 
cosmovisión mesoamericana en comunidades indígenas y originarias itinerantes urbanas contemporáneas, que enriquezcan explicaciones sociohistóricas y comparativas de los procesos culturales, identitarios, simbólicos, lingüísticos, sociales, políticos y económicos inmersos en alternativas interculturales, dirigidas a la construcción de una pedagogía y una educación que reconozca los saberes y las prácticas comunitarias para su comunicación fluida y simétrica con otras tradiciones culturales diferentes y hasta hegemónicas.

\section{Referencias}

Broda, J. (2001). Introducción. En J. Broda y F. BáezJorge (Coords.), Cosmovisión, ritual e identidad de los pueblos indígenas de México (pp. 15-32). México: CONACULTA, Fondo de Cultura Económica.

López Austin, A. (1996). Los mitos del Tlacuache: caminos de la mitología mesoamericana. México: IIA-UNAM.

López, B. (2016). Jek U’nk Kapy: Ritual de iniciación de la comunidad Tamazulápam del Espiritu Santo, Mixe, Oaxaca (Proyecto de tesis para optar al grado de licenciada en Educación Indígena), UPN-Ajusco, México.

Medina, A. (1997). Presentación. En Ciudadanos desde el pueblo. Identidad urbana y religiosidad popular en San Andrés Totoltepec, Tlalpan, México, D. F. (pp. 9-19). México: CONACULTA-UAM/I.

Medina, A. (2000). La textura india de la Ciudad de México. Antropológicas, 17, 5-17.

Medina, A. (2004). Ciclos festivos y rituales en los pueblos originarios de la Ciudad de México: las comunidades de Tláhuac. En P. Yanes et al. (Coords.), Ciudad, Pueblos indígenas y Etnicidad (pp. 151-189). México: Universidad de la Ciudad de México.

Medina, A. (2007). Sistema de cargos y comunidad. Nuevos aportes a una vieja discusión. En Á. Giglia,
C. Garma \& A. De Teresa (Comps.), ¿A dónde va la antropología? (pp. 177-217). México: Ediciones y Gráficos Eón/Tecnigraf Editorial. Colección Biblioteca de Alteridades \#7.

Millán, S. (2007). El cuerpo de la nube. Jerarquía y simbolismo ritual en la cosmovisión de un pueblo huave. México: INAH. Colección Etnografía de los Pueblos Indígenas de México. Serie Estudios Monográficos.

Nemo, T. (2016). Socialización política en medios educativos interculturales. Un estudio de caso xochimilca en medios educativos de extra-edad (Proyecto de interinato de estudiante de maestría del Institut d'Etudes Politiques, Francia). UPN-Ajusco.

Ornelas, G. E. (2010). Cosmovisión en la escuela primaria. Una aportación a la antropología educativa. Cuicuilco. Revista de la ENAH, nueva época, 17(48), 297-321.

Ornelas, G. E. (2007). El ciclo festivo escolar en un pueblo del sur de la Cuenca de México. En A. Medina (Coord.), La memoria negada de la Ciudad de México: sus pueblos originarios (pp. 313-342). México: UNAM/ IIA-UACM/Centro de Estudios sobre la Ciudad.

Ornelas, G. E. (2007a). Narraciones míticas y procesos rituales en la escuela y su entorno. México: UPN.

Ornelas, G. E. (2005). Práctica docente y dinámica cultural en la escuela primaria. México: Cámara de Diputados LIX Legislatura /UPN/ Miguel Ángel Porrúa.

Portal, M. A. (1997). Ciudadanos desde el pueblo. Identidad urbana y religiosidad popular en San Andrés Totoltepec, Tlalpan, México, D.F. México: UAM-I/ CONACULTA.

Turner, V. (1980). La selva de los símbolos. Aspectos del ritual ndembu. México: Siglo XXI. 